\title{
Ship-borne Warning Helicopter Fire Control Radar Antenna Pattern Design and Simulation
}

\author{
Xu Qi \\ Midshipman Brigade of Dalian Naval Academy \\ Dalian, China \\ e-mail: 446982051@qq.com
}

\begin{abstract}
The design and realization of radar antenna direction chart is important in the simulation of radar system. The good design of radar antenna direction chart can improve the rate and reality of radar system simulation. Firstly this paper introduces the traditional antenna direction chart which has two bugs which are normalization and pitch angle. Because of these bugs, this paper introduces antenna direction chart function which is improved and added. This paper simulates the direction chart function. The simulation indicates that through the improved antenna direction, chart is better in pitching and direction and also approaches real radar antenna direction chart.
\end{abstract}

Keywords-radar; antenna; simulate; direction chart

\section{INTRODUCTION}

In simulation of radar and electronic warfare effectiveness evaluation and simulation, the design of the radar antenna is an important part is the foundational, the design of the traditional radar antenna diagram more focus on theoretical level, for the simulation design has certain insufficiency, the improvement is carried on while maintaining the original theory principle, on the basis of closer simulation application, which lays a foundation for the realization of the subsequent simulation.

\section{THE TRADITIONAL ANTENNA PATTERN DESIGN}

In the usual day chart, only the azimuth of the simulation is involved. The orientation of the antenna diagram function using singh function is described as below :

$$
f(\theta)=\frac{1+\cos \theta}{2} \times \frac{\sin (k \sin \theta)}{k \sin \theta}
$$

In this type, $\theta_{b}$ is One-way half power lobe width. And usually achieve good lord sidelobe ratio, the simulation side lobe weighted is given. A requirement for the sidelobe level is $\mathrm{SdB}(\mathrm{S}<50 \mathrm{db})$, the main lobe weighted sidelobe is zero outside parts:

$$
f(\theta)=a \times \frac{|\sin (k \sin \theta)|}{|k \sin \theta|}
$$

Figure 1 draws the sidelobe level of $20 \mathrm{db}$ antenna pattern (horizontal angle value, the unit is degree, ordinate said singh function values).
Teng Zhe

Dept. of Information warfare, Dalian Naval Academy Dalian,China e-mail:37630759@qq.com

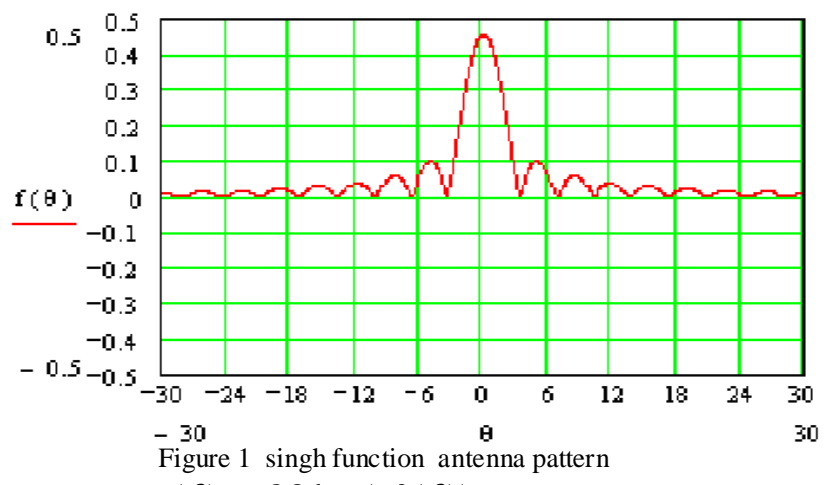

When $y(\theta)=20 \log (f(\theta))$, Antenna pattern is shown in Figure 2 (horizontal angle value, the unit is degree, ordinate said amplitude values) :

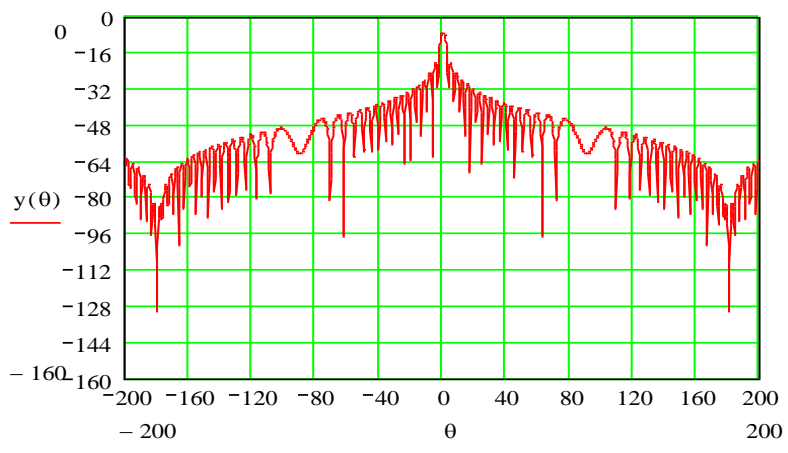

Figure 2 normalized antenna pattern

\section{THE MODIFIED DESIGN OF THE ANTENNA PATTERN}

Because of the type used in antenna pattern model in normalization, and the shortcomings of the pitching Angle and so on, according to the practical characteristics of modern airborne fire control radar antenna and technical parameters, the antenna orientation graph model is improved. This system adopts the single pulse radar system and adopts the four small antenna for receiving and transmitting. Total antenna mouth is evenly divided into four parts, named A, B, C, D, as shown in Figure 3 (in the antenna coordinate system, left A negative right is, is on the negative.) 


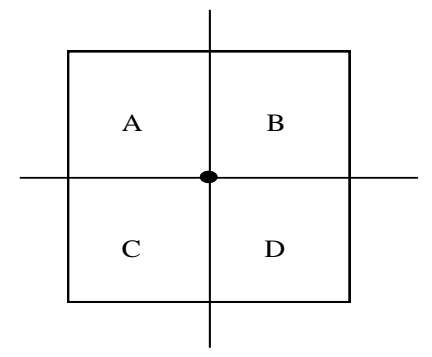

Figure 3 days radar chart

A, B, C, D are four separated points and exactly the same antenna performance. The four common points are the antenna, which is the center of the radar antenna axis and is not only the mechanical shaft radar antenna, but also the radar antenna electrical axis.

Target echo from A, B, C, D four antenna into and poor. After dealing with the addition subtraction and difference (CCM), in the outlet and road:

$$
\Sigma=A+B+C+D
$$

In the poor road:

$$
\begin{aligned}
& \Delta A=(B+D)-(A+C) \\
& \Delta H=(A+B)-(C+D)
\end{aligned}
$$

Corresponding to the antenna pattern functions are:

$$
\begin{aligned}
& \quad f \Sigma(\sigma, \phi)=f A(\sigma, \phi)+f B(\sigma, \phi) \\
& \quad+f C(\sigma, \phi)+f D(\sigma, \phi) \\
& f \Delta A(\sigma, \phi)=f B(\sigma, \phi)+f D(\sigma, \phi) \\
& -f A(\sigma, \phi)-f C(\sigma, \phi) \\
& f \Delta H(\sigma, \phi)=f A(\sigma, \phi)+f B(\sigma, \phi) \\
& -f C(\sigma, \phi)-f D(\sigma, \phi)
\end{aligned}
$$

Four antenna patterns are:

$$
\begin{array}{r}
f A(\sigma, \phi)=G \times a_{1}\left(t \times \sigma_{A}\right) \frac{1+\cos \left(t \times \sigma_{A}\right)}{2} \times \frac{\left|\sin \left(k_{1} \times \sin \left(t \times \sigma_{A}\right)\right)\right|}{\left|k_{1} \times \sin \left(t \times \sigma_{A}\right)\right|} \\
\times a_{2}\left(t \times \phi_{A}\right) \frac{1+\cos \left(t \times \phi_{A}\right)}{2} \times \frac{\left|\sin \left(k_{2} \times \sin \left(t \times \phi_{A}\right)\right)\right|}{\left|k_{2} \times \sin \left(t \times \phi_{A}\right)\right|} \\
f B(\sigma, \phi)=G \times a_{1}\left(t \times \sigma_{B}\right) \frac{1+\cos \left(t \times \sigma_{B}\right)}{2} \times \frac{\left|\sin \left(k_{1} \times \sin \left(t \times \sigma_{B}\right)\right)\right|}{\left|k_{1} \times \sin \left(t \times \sigma_{B}\right)\right|} \times \\
a_{2}\left(t \times \phi_{B}\right) \frac{1+\cos \left(t \times \phi_{B}\right)}{2} \times \frac{\mid \sin \left(k_{2} \times \sin \left(t \times \phi_{B}\right) \mid\right.}{\left|k_{2} \times \sin \left(t \times \phi_{B}\right)\right|} \\
f C(\sigma, \phi)=G \times a_{1}\left(t \times \sigma_{C}\right) \frac{1+\cos \left(t \times \sigma_{C}\right)}{2} \times \frac{\left|\sin \left(k_{1} \times \sin \left(t \times \sigma_{C}\right)\right)\right|}{\left|k_{1} \times \sin \left(t \times \sigma_{C}\right)\right|} \\
\times a_{2}\left(t \times \phi_{C}\right) \frac{1+\cos \left(t \times \phi_{C}\right)}{2} \times \frac{\left|\sin \left(k_{2} \times \sin \left(t \times \phi_{C}\right)\right)\right|}{\left|k_{2} \times \sin \left(t \times \phi_{C}\right)\right|} \\
f D(\sigma, \phi)=G \times a_{1}\left(t \times \sigma_{D}\right) \frac{1+\cos \left(t \times \sigma_{D}\right)}{2} \times \frac{\left|\sin \left(k_{1} \times \sin \left(t \times \sigma_{D}\right)\right)\right|}{\left|k_{1} \times \sin \left(t \times \sigma_{D}\right)\right|} \\
\times a_{2}\left(t \times \phi_{D}\right) \frac{1+\cos \left(t \times \phi_{D}\right)}{2} \times \frac{\left|\sin \left(k_{2} \times \sin \left(t \times \phi_{D}\right)\right)\right|}{\left|k_{2} \times \sin \left(t \times \phi_{D}\right)\right|}
\end{array}
$$

Respectively to mark on the type of parameter:

(1) $G$ is four beam amplitude weighting for the synthesis of a beam.

(2)Perspective transformation for arc factor is $t=\frac{\pi}{180}$ (because of using the pitching Angle and azimuth angle).
(3) $\sigma$ is Antenna azimuth. $\sigma_{A}$ is $\sigma_{A}=\sigma+\sigma_{0}, \sigma_{B}$ is $\sigma_{B}=\sigma-\sigma_{0}, \sigma_{C}$ is $\sigma_{C}=\sigma+\sigma_{0} \quad, \quad \sigma_{D} \quad$ is $\sigma_{D}=\sigma-\sigma_{0}$, Antenna mechanical ax is and space target azimuth is $\sigma$;

The biggest gain of the antenna pattern axis direction and the antenna azimuth is $\sigma_{0}$;

(4) $\phi$ is Antenna pitching angle. For the antenna $\phi_{A}$, a pitching angle is $\phi_{A}=\phi+\phi_{0}$; for the antenna $\phi_{B}$, a pitching angle is $\phi_{B}=\phi-\phi_{0}$; for the antenna $\phi_{C}$, a pitching angle is $\phi_{C}=\phi+\phi_{0}$; for the antenna $\phi_{D}$, a pitching angle is $\phi_{D}=\phi-\phi_{0}$;

Antenna mechanical axis of the pitch angle and space target is $\phi$;

The biggest gain of the antenna pattern direction of the pitch angle and antenna axis is $\phi_{0}$;

(5) A single figure in the direction of the beam singh function coefficient is $k_{1}=\frac{1.3916}{\sin \left(t \times \frac{\theta_{b}}{2}\right)}$, a single beam bearing beam width is $\theta_{b}$;

(6) A single figure in the direction of the beam singh function coefficient is $k_{2}=\frac{1.3916}{\sin \left(t \times \frac{\theta_{a}}{2}\right)}$, a single beam longitudinal beam width is $\theta_{a}$;

(7) Sidelobe antenna beam azimuth weighting factor is $a_{1}(\sigma)$.

$$
a_{1}(\sigma)= \begin{cases}10^{-0.05(S-13.26)} & |\sigma| \geq \frac{\pi}{k_{1}} \\ 1 & |\sigma|<\frac{\pi}{k_{1}}\end{cases}
$$

(8) Antenna beam pitch sidelobe weighted factor is $a_{2}(\sigma)$.

$$
a_{2}(\sigma)= \begin{cases}10^{-0.05(S-13.26)} & |\sigma| \geq \frac{\pi}{k_{2}} \\ 1 & |\sigma|<\frac{\pi}{k_{2}}\end{cases}
$$

This radar simulation sets the main pay disc as $\mathrm{S}=$ $20 \mathrm{db}$, which is a single small antenna beam azimuth with beam width of $\theta_{b}=1.6^{\circ}$, a single small pitch beam with the width of $\theta_{a}=4.2^{\circ}$, composite beam antenna and azimuth half power beam with the width of $2 \sigma=1.6^{\circ}$, composite beam antenna and pitch half power beam with the width of $2 \phi=4.2^{\circ}$. According to the normalized 
gain for one and a half, and the relationship between power point will be more parameters in the normalized antenna and the direction map function

$f \Sigma(\sigma, \phi)=f A(\sigma, \phi)+f B(\sigma, \phi)+f C(\sigma, \phi)+f D(\sigma, \phi)$, namely:

$$
\begin{aligned}
& f \Sigma(0,0)=4 \cdot G \cdot \frac{1+\cos \left(t \sigma_{0}\right)}{2} \cdot \frac{\left|\sin \left(k_{1} \sin \left(t \sigma_{0}\right)\right)\right|}{\left|k_{1} \sin \left(t \sigma_{0}\right)\right|} \cdot \frac{1+\cos \left(t \phi_{0}\right)}{2} \cdot \frac{\left|\sin \left(k_{2} \cdot \sin \left(t \phi_{0}\right)\right)\right|}{\left|k_{2} \cdot \sin \left(t \phi_{0}\right)\right|}=1 \\
& f \Sigma(\sigma, 0)=G \cdot\left(\frac{1+\cos \left(t \phi_{0}\right)}{2} \cdot \frac{\left|\sin \left(k_{2} \cdot \sin \left(t \phi_{0}\right)\right)\right|}{\left|k_{2} \sin \left(t \phi_{0}\right)\right|}\right) \cdot \\
& {\left[a_{1}\left(t\left(\sigma-\sigma_{0}\right)\right) \cdot 1+\cos \left[t\left(\sigma-\sigma_{0}\right)\right] \cdot \frac{\mid \sin \left[k_{1} \cdot \sin \left(t\left(\sigma-\sigma_{0}\right)\right] \mid\right.}{\left|k_{1} \sin \left(t\left(\sigma-\sigma_{0}\right)\right)\right|}\right.} \\
& \left.+a_{1}\left(t\left(\sigma+\sigma_{0}\right)\right) \cdot 1+\cos \left[t\left(\sigma+\sigma_{0}\right)\right] \cdot \frac{\mid \sin \left[k_{1} \cdot \sin \left(t\left(\sigma+\sigma_{0}\right)\right] \mid\right.}{\left|k_{1} \sin \left(t\left(\sigma+\sigma_{0}\right)\right)\right|}\right] \\
& f \Sigma(0, \phi)=G \cdot\left(\frac{1+\cos \left(t \sigma_{0}\right)}{2} \cdot \frac{\left|\sin \left(k_{2} \cdot \sin \left(t \sigma_{0}\right)\right)\right|}{\left|k_{2} \sin \left(t \sigma_{0}\right)\right|} \cdot\right. \\
& {\left[a_{2}\left(t\left(\phi-\phi_{0}\right)\right) \cdot 1+\cos \left[t\left(\phi-\phi_{0}\right)\right] \cdot \frac{\mid \sin \left[k_{1} \cdot \sin \left(t\left(\phi-\phi_{0}\right)\right] \mid\right.}{\left|k_{1} \sin \left(t\left(\phi-\phi_{0}\right)\right)\right|}\right.} \\
& \left.+a_{1}\left(t\left(\phi+\phi_{0}\right)\right) \cdot 1+\cos \left[t\left(\phi+\phi_{0}\right)\right] \cdot \frac{\mid \sin \left[k_{1} \cdot \sin \left(t\left(\phi+\phi_{0}\right)\right] \mid\right.}{\left|k_{1} \sin \left(t\left(\phi+\phi_{0}\right)\right)\right|}\right]
\end{aligned}
$$

Available, amplitude weighting factor $\mathrm{G}=0.475366$, azimuth $=0.610689$, pitching angle $=1.63548$.

\section{ADVANCED ANTENNA PATTERN SIMULATION IMPLEMENTATION}

The parameters obtain above generation into four small antenna antenna pattern, the available antennas and beam:

$$
f \Sigma(\sigma, \phi)=f A(\sigma, \phi)+f B(\sigma, \phi)+f C(\sigma, \phi)+f D(\sigma, \phi)
$$

Antenna azimuth difference beam:

$$
f \Delta A(\sigma, \phi)=f B(\sigma, \phi)+f D(\sigma, \phi)-f A(\sigma, \phi)-f C(\sigma, \phi)
$$

Figures available and direction (angle of 60 degree to 60 degree, the pitching angle of 0 degree) are shown in Figure 4 below (and the solid line shows the antenna beam and dotted line antenna azimuth difference beam with the horizontal angle respectively of 60 degree to 60 degree and the vertical said normalized values) :

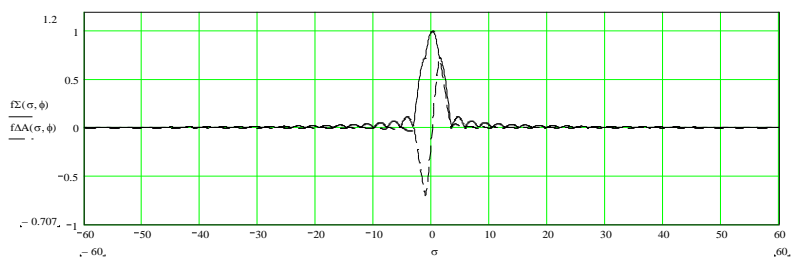

Figure 4 the antenna beam and azimuth difference beam

Available antennas and beam:

$f \Sigma(\sigma, \phi)=f A(\sigma, \phi)+f B(\sigma, \phi)+f C(\sigma, \phi)+f D(\sigma, \phi)$

The antenna elevation difference beam:

$f \Delta H(\sigma, \phi)=f A(\sigma, \phi)+f B(\sigma, \phi)-f C(\sigma, \phi)-f D(\sigma, \phi)$
Available and direction graphs (pitching angle of 60 degree to 60 degree, the azimuth angle of 0 degree) are shown in Figure 5 below (and the solid line shows the antenna beam and dotted line antenna elevation difference beam with horizontal angle of respectively 60 degree to 60 degree, the vertical said normalized values) :

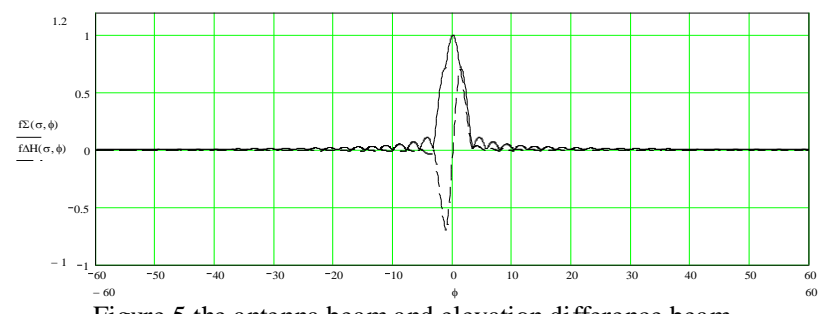

Figure 5 the antenna beam and elevation difference beam

In order to analyze the problem definition, it is convenient to do the following:

Definition 1: meteorological visibility $R_{0}$ weather conditions at the time, the airborne radar maximum detection range.

Definition 2: the earth curvature stadia $R_{D}$ when considering the earth curvature effects of the chase depending on the distance.

As shown in Figure6, in order to target distribution center $M$ as the center of the circle, respectively, to detect distance $R_{0}$, enemy anti-aircraft fire range $d_{m k}$ for the radius, draw arc $d_{m k} \leq R_{0}$ on the surface of the optical axis vertical.

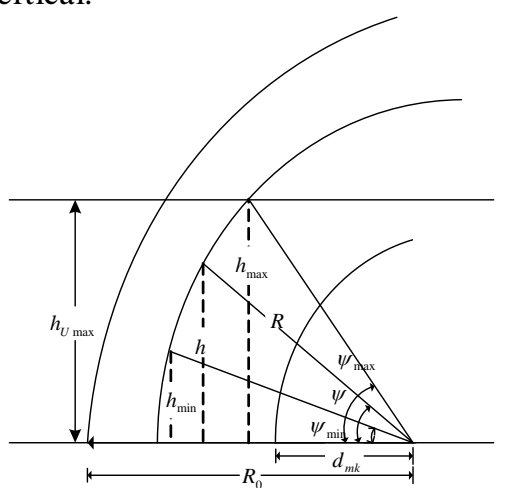

Figure 6. flying height limit range analysis diagram

Obviously, under a given detection range $R \leq R_{0}$, the flight height is $h$, beam angle of depression is $\psi$ which has the maximum and minimum values.

Decision $\psi_{\max }$ factors as follows:

(1) Ship-borne warning helicopter maximum height ceiling $h_{U \max }$

(2) Airborne radar beam load maximum depression angle limits.

Therefore, it can be determined under a given detection range $R \leq R_{0}$, the maximum flying height of the ship-borne warning helicopter $h_{\max }$ and the maximum limits of beam angle of depression $\psi_{\max }$ are as follows: 


$$
\left\{\begin{array}{c}
\psi_{\max }=\min \left(\psi_{U \text { max }}, \arcsin \left(\frac{h_{U \text { max }}}{R}\right)\right) \\
h_{\text {max }}=R \cdot \sin \left(\psi_{\text {max }}\right)
\end{array}\right.
$$

The key factors of the maximum $h$ value are:

Ship-borne warning helicopter maximum height ceiling $h_{U \max }$ (in the case of only $h_{U \max }<R$ discuss).

The key factors of the minimum value $h$ :

(1) Ship-borne warning helicopter minimum safe altitude $h_{U \text { min }}$.

(2) The earth curvature sights $R_{D}=4.12\left(\sqrt{h_{\min }}+\sqrt{h_{m}}\right)$.

(3) Carrying ship of ship-borne warning helicopter effective communication distance $d_{T X}$.

Obviously, given the detection distance $R \leq R_{0}$, the ship-borne warning helicopter to effective detecting target, the minimum altitude should satisfy:

$$
h_{\min } \geq h_{1}=\left(\frac{R}{4.12}-\sqrt{h_{m}}\right)^{2}
$$

In formation against the distance $d_{w m}$ between the ship and ship-borne, warning helicopter for effective communication, the minimum altitude ship-borne warning helicopter should satisfy:

$$
h_{\min } \geq h_{2}=\left(\frac{d_{w m}}{4.12}-\sqrt{h_{w}}\right)^{2}
$$

$d_{w m} \leq d_{T X}, h_{w}$ carrier communication antenna height $(/ \mathrm{m})$.

Therefore, itcan be determined under a given detection range $R \leq R_{0}$, minimum flying height of shipborne warning helicopter $h_{\min }$ and minimum limits of beam Angle of depression $\psi_{\min }$ are as follows:

$$
\left\{\begin{array}{c}
h_{\min }=\max \left(h_{U \min }, h_{1}, h_{2}, R \sin \psi\right) \\
\psi_{\min }=\arcsin \left(\frac{h_{\min }}{R}\right)
\end{array}\right.
$$

\section{THE SIMULATION ANALYSIS}

(1) set the simulation conditions

According to the actual combat of battlefield situation, set up the simulation conditions are as follows:

$$
\begin{gathered}
h_{U \max }=3500 \mathrm{~m} \quad, \quad d_{T X}=150 \mathrm{~km} \square 80 \mathrm{~km} \\
h_{w}=25 \mathrm{~m}, h_{m}=16 \mathrm{~m}, h_{U \text { min }}=500 \mathrm{~m}
\end{gathered}
$$

Model detection 'of the simulation results of characterization in a given distance $R$, the distance between the enemy formation $d_{w m}$, the height of ship-

\begin{tabular}{|c|c|c|c|c|c|c|c|c|}
\hline \multirow{2}{*}{$\begin{array}{r}\text { Search } \\
\text { arameters } \\
\text { range }\end{array}$} & \multicolumn{2}{|c|}{$\mathrm{D}_{1}$} & \multicolumn{2}{|c|}{$\mathrm{D}_{2}$} & \multicolumn{2}{|c|}{$\mathrm{D}_{3}$} & \multicolumn{2}{|c|}{$\mathrm{D}_{4}$} \\
\hline & $\left(h_{\min } / h_{\max }\right)$ & $\left(\psi_{\min } / \psi_{\max }\right)$ & $\left(h_{\min } / h_{\max }\right)$ & $\left(\psi_{\min } / \psi_{\max }\right)$ & $\left(h_{\min } / h_{\max }\right)$ & $\left(\psi_{\min } / \psi_{\max }\right)$ & $\left(h_{\min } / h_{\max }\right)$ & $\left(\psi_{\min } / \psi_{\max }\right)$ \\
\hline $\mathrm{d}_{1}$ & $(582.1 / 3500)$ & $(0.83 / 5.02)$ & $(500 / 3500)$ & $(0.72 / 5.02)$ & $(500 / 3500)$ & $(0.72 / 5.02)$ & $(500 / 3500)$ & $(0.72 / 5.02)$ \\
\hline $\mathrm{d}_{2}$ & $(582.1 / 3500)$ & $(0.67 / 4.01)$ & $(500 / 3500)$ & $(0.57 / 4.01)$ & $(500 / 3500)$ & $(0.57 / 4.01)$ & $(500 / 3500)$ & $(0.57 / 4.01)$ \\
\hline $\mathrm{d}_{3}$ & (582.1/3500) & $(0.56 / 3.34)$ & $(500 / 3500)$ & $(0.48 / 3.34)$ & $(500 / 3500)$ & $(0.48 / 3.34)$ & $(500 / 3500)$ & $(0.48 / 3.34)$ \\
\hline $\mathrm{d}_{4}$ & $(582.1 / 3500)$ & $(0.48 / 2.87)$ & $(500 / 3500)$ & $(0.41 / 2.87)$ & $(500 / 3500)$ & $(0.41 / 2.87)$ & $(500 / 3500)$ & $(0.41 / 2.87)$ \\
\hline $\mathrm{d}_{5}$ & $(582.1 / 3500)$ & $(0.42 / 2.51)$ & $(500 / 3500)$ & $(0.36 / 2.51)$ & $(500 / 3500)$ & $(0.36 / 2.51)$ & $(500 / 3500)$ & $(0.36 / 2.51)$ \\
\hline $\mathrm{d}_{6}$ & $(582 / 3500)$ & $(0.37 / 2.23)$ & $(500 / 3500)$ & $(0.32 / 2.23)$ & $(500 / 3500)$ & $(0.32 / 2.23)$ & $(500 / 3500)$ & $(0.32 / 2.23)$ \\
\hline $\mathrm{d}_{7}$ & $(582 / 3500)$ & $(0.33 / 2.00)$ & $(500 / 3500)$ & $(0.27 / 2.00)$ & $(500 / 3500)$ & $(0.27 / 2.00)$ & $(500 / 3500)$ & $(0.27 / 2.00)$ \\
\hline$d_{8}$ & $(582 / 3500)$ & $(0.30 / 1.82)$ & $(515.2 / 3500)$ & $(0.27 / 1.82)$ & $(515.2 / 3500)$ & $(0.27 / 1.82)$ & $(515.2 / 3500)$ & $(0.27 / 1.82)$ \\
\hline $\mathrm{d}_{9}$ & $(631 / 3500)$ & $(0.30 / 1.67)$ & $(631.3 / 3500)$ & $(0.30 / 1.67)$ & $(631.3 / 3500)$ & $(0.30 / 1.67)$ & $(631.3 / 3500)$ & $(0.30 / 1.67)$ \\
\hline$d_{10}$ & $(759 / 3500)$ & $(0.33 / 1.54)$ & (759.1/3500) & $(0.33 / 1.54)$ & (759.1/3500) & $(0.33 / 1.54)$ & (759.1/3500) & $(0.33 / 1.54)$ \\
\hline
\end{tabular}
borne warning helicopter detecting $h$, the scope of pitching Angle of the beam $\psi$. The calculation results are shown in Table 2.

Among them:

TABLE I SHIP-BORNE W ARNING HELICOPTER SEARCH PARAMETERS

(2) the use of Table 1

a. to determine the use of airborne radar beam pitching Angle.

b. according to the battlefield, situation is used to target detection distance. c. To determine the ship-borne warning helicopter flew to the task area, the distance between the enemy formations.

(3) according to the combat mission against distance, to find that the distance required by the requirement of 
ship-borne warning helicopter flight height and pitch range of the beam.

(4) the altitude in the table and the use of the visual axis bow angle range and control method.

If you want to always stay in determining the maximum effective detection range of target search, by choosing the corresponding maximum flying height in pitching angle of the beam. When it comes to lower altitude and wants to keep the maximum effective detection range, it should increase the pitching angle of the beam, the visual axis should be brought up. When raising altitude and the maximum effective detection range should be kept, pitching angle of the beam,should be reduced and the visual axis should be brought to the press.

\section{CONCLUSION}

Ship-borne warning helicopter should determine its operational method of use in a fleet air defense combat, the design method can effectively calculate the airspace cumulative detection probability that the ship-borne warning helicopter detect on a particular incoming target a location, and it can provide the commander with no less than the one target probability search the target distance, so as to determine the scope of the flight altitude and pitching angle of the beam and provides reference for ship-borne warning helicopter fleet air defense combat use.

\section{REFERENCE}

[1] Galay Barbarosoglu, Linet Ozdamar, Ahmel Cevik.An Interactive Appmach of Hierarchical Analysis of Helicopter Logistics in Disaster Relief Operations[J]. European Journal of Operational Research, 2002 : 118-133

[2] WANG Hong-jun, CHI Zhong-xian.Shipboard hard and soft weapon anti-missile decision-makingoptimization based on
collaboration[J], Control and Decision, 2007, 22 (3) : 299303

[3] Liang Bo, Duan Ran.Fire distribution model based on feasible direction algorithm $[\mathrm{J}]$, Command Information System and Technology, 2013,4 (2) :30-32

[4] L Ozdamar . Emergency logistics planning in natural disasters[J]. Annal of Operation Research, 2004, 129(11) : 218-219.

[5] Sarit Kraus.Negotiation and cooperation in mulfi-ageat environments.Artificial Intelligence. 1997 : 79-97

[6] HUANG Haixin, WANG Ding wei.English auction dynamic model based on fuzzy game[J], Information and Control,2012,41(4):509-513

[7] GAO Zhi hua,CHEN Jian,WEN Jianguo.Optimal Firing Distribution of the Air Defense Based on Genetic Algorithm[J],Computer Digital Engineering,2013,44 (5) :733735

[8] Shao Qiu feng, Ma ya long, hu jin chuan.Applied research for fuzzy composite operators in operational alternatives integrated evaluation[J],Journal of Academy of Armored Force Engineering, 2004, 18 (12) : 41-43

[9] LI Zi fen, LI Xiang min, CHEN Jin zhu.Dynamic joint fire distribution method based on decentralized cooperative auction algorithm[J],Fire Control \& Command Control , 2012,37 (11) :50-52

[10] NIAN Song lei, YAN Jian gang, CHEN Rong.The optimization method of antiship missile fire distribution based on dynamic programming[J],Ship Science And Technology, 2012,34 (7) $: 110-113$

[11] MA Liang, QING Zuo-sheng, ZHANG Lin.Formation comprehensive cooperative air defense multi-stage decision optimization modeling[J], Tactical Missile Technology, 2013,9 (5) :25-28

[12] LIU Wei dong, JIANG Qing shan, LI Yong.Fire distribution of the network centric ship-to-air missile based on earlier damage[J],Ship Science And Technology, 2011,33 (2) :98-101

[13] CHEN Guo sheng, JIA Zi ying.Research on coordinated air defense firepower distribution model for warships $[\mathrm{J}]$, Command Control\& Simulation , 2011,33 ( 12$)$ :13-15 